\title{
Cerebral tissue oxygenation index in very premature infants
}

\author{
G Naulaers, G Morren, S Van Huffel, P Casaer, H Devlieger
}

Arch Dis Child Fetal Neonatal Ed 2002;87:F189-F192

See end of article for authors' affiliations .....................

Correspondence to: Dr Naulaers, Neonatal Intensive Care Unit, University Hospital 'UZ Gasthuisberg, Herestraat 49, 3000 Leuven, Belgium; gunnar.naulaers@uz. kuleuven.ac.be

Accepted 14 May 2002

\begin{abstract}
Aim: To describe normal values of the cerebral tissue oxygenation index (TOI) in premature infants. Methods: TOI was measured by spatially resolved spectroscopy in preterm infants on the first 3 days of life. Infants with an abnormal cranial ultrasound were excluded. Other simultaneously measured variables were $\mathrm{PaO}_{2}, \mathrm{PaCO}_{2}, \mathrm{pH}$, mean arterial blood pressure, heart rate, haemoglobin, glycaemia, and peripheral oxygen saturation.

Results: Fifteen patients with a median postmenstrual age of 28 weeks were measured. There was a significant increase in median TOl over the first 3 days of life: $57 \%$ on day $1,66.1 \%$ on day 2 , and $76.1 \%$ on day 3 . Multiple regression analysis showed no correlation between TOI and postmenstrual age, peripheral oxygen saturation, mean arterial blood pressure, $\mathrm{PaO}_{2}, \mathrm{PaCO}_{2}$, and haemoglobin concentration.

Conclusion: Cerebral TOI increases significantly in the first 3 days of life in premature babies. This increase probably reflects the increase in cerebral blood flow at this time.
\end{abstract}

$\mathrm{S}$ patially resolved spectroscopy is a new method, using near infrared spectroscopy (NIRS), for measuring cerebral haemoglobin oxygen saturation. The tissue oxygenation index (TOI) is measured with a light detector with three sensors placed at different distances from the near infrared light source. If light with a given intensity is sent into tissue, it is attenuated because of "scatter" loss and "absorption" loss. If the distance between the light source and the sensor is large enough, the isotropy of scatter distribution becomes so homogeneous that the scatter loss is the same at the three sensors. Therefore any differences in intensity measured at the three different sensors can be interpreted as differences in absorption loss. Thus the local absorption change can be seen as a function of the distance between the light source and the three sensors. With this information, an oxygenation index can be calculated using a previously reported algorithm. ${ }^{12}$

Several research groups have measured TOI in healthy adults with different NIRS instruments, ${ }^{3-7}$ and no differences in TOI values between the right and left forehead have been found. To our knowledge, no normal cerebral TOI values in the first days of life have been reported for premature babies (less than 30 weeks gestation). In this study, we measured TOI on days 1,2 , and 3 to obtain "normal" values and to investigate changes with postnatal age.

\section{PATIENTS AND METHODS}

Fifteen patients with a postmenstrual age of less than 31 weeks were included. The median postmenstrual age was 28 weeks (range 25-30). A brain ultrasound was performed on all patients before measurements were started. Exclusion criteria were an abnormal brain ultrasound before the TOI measurement and severe pulmonary hypertension as evidenced by echocardiography and/or congenital malformations.

An NIRO 300 (Hamamatsu Phototonics K.K., Tokyo, Japan) was used for spatially resolved spectroscopy. The optode was placed at the right frontoparietal side with the sensors at $4 \mathrm{~cm}$ distance. All patients were measured within 6 hours of birth for at least 30 minutes. The second and third measurements were performed 24 and 48 hours later. The specific variable measured was TOI.
The simultaneously studied electrocardiogram, pulse rate and peripheral oxygen saturation (beat to beat, on a Nelcor-2000 monitor), and mean arterial blood pressure (Siemens, Sirecus) were recorded in an analogous way with a sampling frequency of $100 \mathrm{~Hz}$ by the data acquisition system Codas (Dataq Instruments, Akron, Ohio, USA) and stored in a PC. The NIRO-300 signals are digital and recorded with a sampling rate of $6 \mathrm{~Hz}$. They are converted into analogue signals with a sample and hold function before their introduction into the Codas system. $\mathrm{PaCO}_{2}, \mathrm{PaO}_{2}, \mathrm{pH}$, glycaemia, haemoglobin, and percentage of fetal haemoglobin were measured in an arterial blood sample on a blood gas analyser (Radiometer, Copenhagen, Denmark) before and after the measurement. The same author (GN) performed all the measurements.

The median and 95\% confidence interval (CI) was calculated for the TOI values if the peripheral oxygen saturation did not change more than $5 \%$. The median and $95 \%$ CI of heart rate, peripheral oxygen saturation, mean arterial blood pressure, $\mathrm{PaO}_{2}, \mathrm{PaCO}_{2}$, haemoglobin, percentage of fetal haemoglobin, and glycaemia over this period were also calculated. A Kolmogorov-Smirnov test was used to test for a normal distribution of the TOI for the whole group and the three subgroups (different days). The median and 95\% CI of the standard deviation on each TOI measurement was calculated to show the variability in this parameter.

Analysis of variance was applied to see if there was a significant difference between TOI and postnatal age. The Student-Newman-Keuls test was used for all pairwise comparisons. $p<0.05$ was considered significant. Multiple regression analysis was used to detect significant effects of the other variables on TOI.

\section{RESULTS}

Fifteen patients were studied during the first 3 days of life. The mean (SD) birth weight was 1053 (395) g, and the mean (SD)

Abbreviations: NIRS, near infrared spectroscopy; TOI, tissue oxygenation index 
Table 1 Median and 95\% confidence interval for tissue oxygenation index, THI, mean arterial blood pressure, peripheral oxygen saturation, $\mathrm{PaO}_{2}, \mathrm{PaCO}_{2}$, haemoglobin, and fetal haemoglobin on days 1,2 , and 3 .

\begin{tabular}{|c|c|c|c|c|}
\hline & Day 1 & Day 2 & Day 3 & $\mathrm{p}$ Value \\
\hline TOI (\%) & 57 (54 to 65.7) & $66.1(61.9$ to 82.2$)$ * & $76.1(67.1 \text { to } 80.1)^{*}$ & $<0.05$ \\
\hline THI (au) & 37.8 (15 to 64.1$)$ & 31.1 (12.3 to 51.2$)$ & 23.7 (14 to 61.6$)$ & 0.49 \\
\hline $\mathrm{MABP}(\mathrm{mm} \mathrm{Hg})$ & 32 (30.7 to 39.6$)$ & 36 (34 to 42.1$)$ & $41(37.4 \text { to } 45)^{*}$ & 0.03 \\
\hline Saturation $(\%)$ & $96.3(94.5$ to 98$)$ & $96(92$ to 98.5$)$ & $95.1 \quad(92.1$ to 97.5$)$ & 0.44 \\
\hline $\mathrm{PaO}_{2}(\mathrm{~mm} \mathrm{Hg})$ & $62(57$ to 96$)$ & 55 (49.9 to 72$)$ & $57.5(53.3$ to 65$)$ & 0.067 \\
\hline $\mathrm{PaCO}_{2}(\mathrm{~mm} \mathrm{Hg})$ & $31(26$ to 38.1$)$ & $45(37.9 \text { to } 49.4)^{*}$ & $36.5(31.6 \text { to } 43)^{*}$ & $<0.001$ \\
\hline $\mathrm{Hb}(\mathrm{g} / \mathrm{dl})$ & $14.1 \quad(12.3$ to 15.5$)$ & 13 (11.6 to 15.2$)$ & $14.1 \quad(12.3$ to 15.5$)$ & 0.052 \\
\hline Fetal Hb (\%) & 86.7 (81.8 to 91.6$)$ & 85.5 (72.2 to 90$)$ & $69.7(56.9 \text { to } 82.4)^{* *}$ & 0.006 \\
\hline
\end{tabular}

* Significant difference $(p<0.05)$ from day 1 (analysis of variance).

$* *$ Significant difference $(\mathrm{p}<0.08)$ from day 2 (analysis of variance)

TOI, Tissue oxygenation index; THI, tissue haemoglobin index; MABP, mean arterial blood pressure; $\mathrm{Hb}$, haemoglobin.

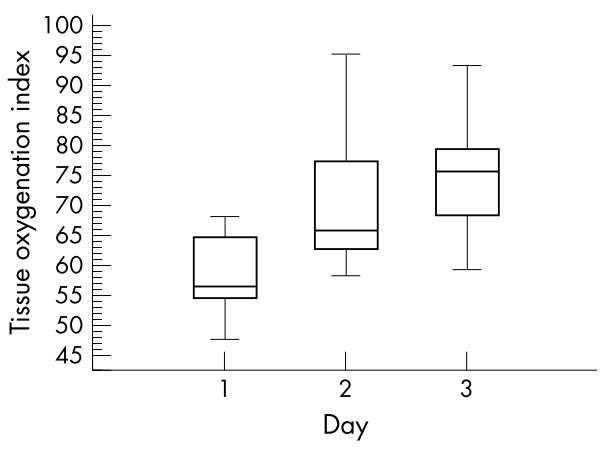

Figure 1 Box and whisker plot showing the median and interquartile range for the tissue oxygenation index $(\%)$ measured on days 1,2 , and 3 . The increase from day 1 to day 2 and from day 1 to day 3 was significant $(p<0.05)$.

head circumference was $24.8(2.5) \mathrm{cm}$. All patients had a normal ultrasound of the brain before measurement on day 1 . Further follow up of the ultrasound on days 3, 5, and 7 showed an intraventricular haemorrhage grade I or II in seven of the 15 patients. However, no posthaemorrhagic hydrocephalus or intraventricular haemorrhage grade III or IV was noted. One patient developed cystic periventricular leucomalacia three weeks later. Seven patients developed retinopathy of prematurity grade III, and three of these patients needed cryotherapy. Psychomotor follow up was normal in 10 patients at the age of 6 months.

Figure 1 shows the TOI values. The median TOI was $57 \%$ (95\% CI 54 to $65.7 \%$ ) on day 1, 66.1\% (95\% CI 61.982.2\%) on day 2 , and $76.1 \%$ (95\% CI 67.8 to $80.1 \%$ ) on day 3. The mean standard deviation of the TOI values was $2.2 \%$ (95\% CI $2.4 \%$ ). There was a normal distribution for the whole group as well as for the three different subgroups (Kolmogorov-Smirnov test for normal distribution, $p>0.2$ ). Analysis of variance showed a significant difference $(\mathrm{p}<0.001)$ between TOI and postnatal age. A Student-Newman-Keuls test for all pairwise comparisons showed that there was a significant increase in TOI from day 1 to day 2 and from day 1 to day 3, but not from day 2 to day 3 .

Table 1 shows the different variables. To test the effect of oxygenation, analysis of variance was performed to find an eventual difference in peripheral oxygen saturation for the different days. No significant difference was found $(p=0.44)$. A significant $(p=0.003)$ increase in blood pressure from day 1 to day 3 was found. The increase in blood pressure from day 1 to day 2 was not significant. There was also a significant difference in $\mathrm{PaCO}_{2}$ on the different days. Mean $\mathrm{PaCO}_{2}$ on day 1 was $31 \mathrm{~mm} \mathrm{Hg}$, on day $245 \mathrm{~mm} \mathrm{Hg}$, and on day $336.5 \mathrm{~mm} \mathrm{Hg}$. This is a significant difference $(p<0.001)$ between the three days, but there was an increase from day 1 to 2 and a decrease from day 2 to 3. There was no significant difference in $\mathrm{PaO}_{2}$, bicarbonate, and $\mathrm{pH}$ on the different days. Haemoglobin concentra- tion was $14.1 \mathrm{~g} / \mathrm{dl}$ on day $1,13 \mathrm{~g} / \mathrm{dl}$ on day 2 , and $14.1 \mathrm{~g} / \mathrm{dl}$ on day 3 . The percentage of fetal haemoglobin was only measured in eight patients. It was $86.7 \%$ on day $1,88.5 \%$ on day 2 , and $69.7 \%$ on day 3 . This significant decrease on day 3 is explained partly by the fact that these patients had received a blood transfusion.

Multiple regression analysis showed an increase in TOI only from day 1 to day $3(\mathrm{p}<0.05)$. No correlation between TOI and blood pressure, $\mathrm{PaCO}_{2}$, peripheral oxygen saturation, haemoglobin concentration, fetal haemoglobin, glycaemia or postmenstrual age was found with multiple regression analysis. No correlation was found between TOI or the standard deviation of TOI and head circumference.

No relation was found between TOI and intraventricular bleeding, periventricular leucomalacia, retinopathy, or abnormal psychomotor follow up.

\section{DISCUSSION}

NIRS is a non-invasive method for measuring oxygenated and deoxygenated haemoglobin and derived values of brain oxygenation, cerebral blood flow, and cerebral blood volume. Until now, it has been used only in research because it is very sensitive to movement artefacts. Furthermore, it does not provide absolute values, only values relative to the starting point in a continuous way. TOI, in contrast, is an absolute value and can be measured on different occasions in the same patient. Although in this study TOI was determined in very premature babies and their head circumference was small, very stable TOI values with a mean standard deviation of $2.2 \%$ were obtained from measurements taken over at least 30 minutes.

Whether TOI mainly reflects cerebral venous saturation is still under discussion. Several studies report TOI values obtained in healthy adult volunteers. ${ }^{3-7}$ Quaresima et al ${ }^{3}$ compared TOI with cerebral venous oxygen saturation, measured by NIRS (NIRO 300, Hamamatsu), and concluded that TOI mainly reflected saturation of the intracranial venous compartment. Two studies using different NIRS instruments also found a correlation, but between TOI and jugular bulb oximetry. ${ }^{89}$ Other studies comparing jugular bulb oximetry with TOI did not find any correlation. ${ }^{10-14}$ Al-Rawi et al ${ }^{15}$ measured TOI in 60 patients undergoing endarterectomy. They found a significant correlation between TOI and flow velocity, measured by transcranial Doppler of the ipsilateral middle cerebral artery. The change in TOI was predominantly associated with internal carotid artery clamping, and a change during external carotid artery clamping was only seen if there was also a change in blood pressure. The sensitivity of TOI to intracranial and extracranial changes, when there were no blood pressure changes or an extracranial to intracranial anastomosis, was $87.5 \%$ and $0 \%$ respectively and the specificity was $100 \%$ and $0 \%$ respectively. They conclude that TOI predominantly measures intracranial changes. Another important finding was that a decrease in TOI is much more 
important than the absolute value. Different patients had different absolute values, but they all showed a large decrease when the internal carotid artery was clamped (mean (SD) difference in TOI $=-9.4(7.1) \%$ ).

In adults, normal TOI values have been reported to range from $65 \%$ to $85 \%{ }^{3-7}$ Teller et al $^{16}$ reported TOI values for the liver and brain during feeding in 25 infants, aged 1-54 days (mean 15.2 ) with a postmenstrual age of 29-40 weeks (mean 34 weeks and 1 day) and a weight of 1400-3365 g (mean 2385). Mean (SD) TOI of the brain was $62.1(9.7) \%$, which is lower than the values reported in adults. They are comparable to our results on day $1(59.2(6.4) \%)$, although TOI values on days 2 and 3 were higher and more compatible with the results in adult volunteers. Dani et $a l^{17}$ described mean cerebral oxygen saturation during caffeine (group A) and aminophylline (group B) treatment in 40 infants with a mean (SD) gestational age of 30.4 (3) weeks in group A and 29.4 (1.4) weeks in group B. The postnatal age was 19 (13) days in group A and 22 (13) days in group B. The mean cerebral oxygen saturation before and after treatment ranged from $68.5(9.1) \%$ to $70.1(6.6) \%$ in group A and from $64.6(13) \%$ to 68.1 (15)\% in group B. These results are comparable to our results on days 2 and 3.

There are different plausible explanations for the increase in TOI found in this study during the first 3 days of life. An increase in oxygenation was ruled out by including only the results obtained while peripheral oxygen saturation remained stable. Also arterial oxygen content did not change significantly on the different days. Haemoglobin content decreased from day 1 to day 3, but no significant correlation was found with TOI. Fetal haemoglobin content decreased significantly, but again no correlation was found with TOI. Further studies are indicated, as fetal haemoglobin was measured in only eight patients.

One explanation for the increase in TOI is an increase in cerebral blood flow, as has been found previously. ${ }^{6}{ }^{18}$ Meek et al ${ }^{19}$ described a significant increase in cerebral blood flow during the first 72 hours in premature infants, independent of mean arterial blood pressure, $\mathrm{PaCO}_{2}$ and packed cell volume. An important bias in our study is the low $\mathrm{PaCO}_{2}$ values on the first day. This could partly explain the lower cerebral blood flow and hence the lower TOI values, although we found no correlation between $\mathrm{PaCO}_{2}$ and TOI. In adults no correlation was found between TOI and $\mathrm{PaCO}_{2} \cdot{ }^{12}{ }^{14} \mathrm{~A}$ decrease in cerebral blood flow during hypocapnia is well described in neonates. ${ }^{20}$ The combination of low $\mathrm{PaCO}_{2}$ with low blood pressure may explain a lower cerebral blood flow and hence a greater oxygen uptake with a lower venous saturation. Measurements of the venous oxyhaemoglobin saturation confirm this hypothesis. Venous oxyhaemoglobin saturation can be measured by NIRS with partial jugular venous occlusion. Yoxall et $a^{l^{21}}$ validated this technique with co-oximetry of jugular bulb blood obtained during cardiac catheterisation in infants and young children. When cerebral blood flow and cerebral venous oxyhaemoglobin saturation are measured, cerebral oxygen consumption can be calculated. An increase in cerebral oxygen consumption was found with advancing gestational age. ${ }^{22}$ Wardle et al ${ }^{23}$ found an increase in the fractional oxygen extraction with a decrease in $\mathrm{PCO}_{2}$ and an increase during blood transfusion. This may explain the lower TOI in our patients on the first day when there was a significantly lower $\mathrm{PaCO}_{2}$.

We found no relation between TOI and neurological complications or retinopathy, but the measurements were limited to half an hour a day. The neurological and ophthalmic complications may be related to hypocapnia on the first day. In preterm babies, a statistical correlation has been found in different studies between hypocapnia and brain damage and later developmental deficit. ${ }^{20}$

Yoxall et $a l^{21}$ suggest that measurement of cerebral oxygenation gives more information than measurement of cerebral blood flow for the prevention of cerebral hypoxia and ischaemia. Measurements of venous oxyhaemoglobin saturation, cerebral oxygen consumption, and cerebral fractional oxygen extraction can be performed with NIRS. ${ }^{21-23}$ However, measurement of cerebral venous oxyhaemoglobin saturation is still difficult and cannot be performed continuously. TOI is a noninvasive parameter for measurement of cerebral oxygenation and may be useful for continuous monitoring of venous oxyhaemoglobin saturation. Although several validation studies have been carried out in adults, further studies in animals need to be performed to study the relation between TOI, venous jugular saturation, and cerebral blood flow. In neonates, further studies are needed to determine the relation between TOI and venous oxyhaemoglobin saturation as measured by partial jugular venous occlusion. Further clinical studies should attempt to elucidate the variation in TOI over several days. These longer lasting measurements may be able to detect the relation between periods of low cerebral oxygenation and neurological complications such as periventricular leucomalacia. As the measurement of TOI is less sensitive to movement artefacts, these studies may result in a new way to detect and prevent severe cerebral ischaemia by continuous monitoring of cerebral oxygenation.

In conclusion, measurement of TOI in premature infants is a new non-invasive method for measuring aspects of cerebral brain oxygenation. An increase in TOI over the first 3 days of life was found in 15 premature babies. This increase in TOI may reflect an increase in cerebral blood flow during this time.

\section{ACKNOWLEDGEMENTS}

This research was sponsored by the Belgian Programme IVAP V-10-29, the Flemish GOA project MEFISTO-666, and the KV Leven project IDO/99/03.

\section{Authors' affiliations}

G Naulaers, P Casaer, H Devlieger, Department of Paediatrics,

University Hospital Leuven, Belgium

G Morren, S Van Huffel, ESAT/SISTA-COSIC, Department of Electrical Engineering, KU Leuven, Belgium

\section{REFERENCES}

1 Matcher J, Kirckpatrick P, Nahid K, et al. Absolute quantification methods in tissue near infrared spectroscopy. Proc SPIE 1995:2389:486-95.

2 Suzuki S, Takasaki S, Ozaki T, et al. A tissue oxygenation monitor using NIR spatially resolved spectroscopy. Proc SPIE 1999;3597:582-92.

3 Quaresima V, Sacco S, Totaro R, et al. Noninvasive measurement of cerebral hemoglobin oxygen saturation using two near infrared spectroscopy approaches. J Biomed Opt 2000;5:201-5.

4 Samra S, Dorj P, Zelenoek GB, et al. Cerebral oximetry in patients undergoing carotid endarterectomy under regional anesthesia. Stroke 1996;27:49-55

5 Misra M, Stark J, Dujovny M, et al. Transcranial cerebral oximetry in random normal subjects. Neurol Res 1998;20:137-41.

6 Carlin RE, McGraw DJ, Calimlim JR, et al. The use of near-infrared cerebral oximetry in awake carotid endarterectomy. J Clin Anesth 1998;10:109-13

7 Nielsen HB, Boushel R, Madsen P, et al. Cerebral desaturation during exercise reversed by $\mathrm{O}_{2}$ supplementation. Am J Physiol 1999:277:H1045-52.

8 Daubeney PEF, Pilkington SN, Janke E, et al. Cerebral oxygenation measured by near-infrared spectroscopy: comparison with jugular bulb oximetry. Ann Thorac Surg 1996;61:930-4.

9 Sapire KJ, Gopinath SP, Farhat G, et al. Cerebral oxygenation during warming after cardiopulmonary bypass. Crit Care Med 1997:25: 1655-62

10 Buunk G, van der Hoeven G, Meinders AE. A comparison of near-infrared spectroscopy and jugular bulb oximetry in comatose patients resuscitated from a cardiac arrest. Anaesthesia 1998;53:13-19.

11 Minassian AT, Poirier N, Pierrot $M$, et al. Correlation between cerebral oxygen saturation measured by near-infrared spectroscopy and jugular oxygen saturation in patients with severe closed head injury Anesthesiology 1999;91:985-90.

12 Germon TJ, Young AER, Manara AT, et al. Extracerebral absorption of near infrared light influences the detection of increased cerebral oxygenation monitored by near infrared spectroscopy. J Neurol Neurosurg Psychiatry 1995;58:477-9.

13 Lewis SB, Myburgh JA, Thornton EL, et al. Cerebral oxygenation monitoring by near-infrared spectroscopy is not clinically useful in patients with severe closed-head injury: a comparison with jugular venous bulb oximetry. Crit Care Med 1996;24:1334-8. 
14 Ali MS, Harmer M, Vaughan RS, et al. Spatially resolved spectroscopy (NIRO-300) does not agree with jugular bulb oxygen saturation in patients undergoing warm bypass surgery. Can J Anaesth 2001:48:497-501.

15 Al-Rawi PG, Smielewski P, Kirckpatrick PJ. Evaluation of a near-infrared spectrometer (NIRO 300) for the detection of intracranial oxygenation changes in the adult head. Stroke 2001;32:2492-500.

16 Teller J, Schwendener K, Wolf $M$, et al. Continuous monitoring of liver oxygenation with near infrared spectroscopy during naso-gastric tube feeding in neonates. Schweiz Med Wochenschr 2000;130:652-6.

17 Dani C, Bertini G, Reali MF, et al. Brain hemodynamic changes in preterm infants after maintenance dose caffeine and aminophylline treatment. Biol Neonate 2000;78:27-32.

18 Hueber DM, Franceschini MA, Ma HY, et al. Non-invasive and quantitative near-infrared haemoglobin spectrometry in the piglet brain during hypoxic stress, using a frequency-domain multidistance intrument. Phys Med Biol 2001;46:41-62.
19 Meek JH, Tyszczuk L, Elwell CE, et al. Cerebral blood flow increases over the first three days of life in extremely preterm neonates. Arch Dis Child Fetal Neonatal Ed 1998;78:F33-7.

20 Greisen G, Vannuci RC. Is periventricular leucomalacia a result of hypoxic-ischaemic injury? Hypocapnia and the preterm brain. Biol Neonate $2001 ; 79: 194-200$

21 Yoxall CW, Weindling AM, Dawani NH, et al. Measurement of cerebral venous oxyhemoglobin saturation in children by near-infrared spectroscopy and partial jugular venous occlusion. Pediatr Res 1995:38:319-23.

22 Yoxall CW, Weindling AM. Measurement of cerebral oxygen consumption in the human neonate using near infrared spectroscopy: cerebral oxygen consumption increases with advancing gestational age. Pediatr Res 1998;44:283-90.

23 Wardle SP, Yoxall CW, Weindling AM. Determinants of cerebral fractional oxygen extraction using near infrared spectroscopy in preterm neonates. J Cereb Blood Flow Metab 2000;20:272-9.

\section{Rapid responses}

Letters on the following Archives of Disease in Childhood Fetal and Neonatal Edition papers have been published recently as rapid responses on the $A D C$ website. To read these letters visit www.archdischild.com and click on "Read eletters"

Posthaemorrhagic ventricular dilatation in the premature infant: natural history and predictors of outcome. B P Murphy, T E Inder, V Rooks, et al. Arch Dis Child Fetal Neonatal Ed 2002;87:F37-F41.

Neonatal shaken baby syndrome: an aetiological view from Down under. A N Williams, R Sunderland. Arch Dis Child Fetal Neonatal Ed 2002;87:F29-F30

If you would like to post an electronic response to these or any other articles published in the journal, please go to the website, access the article in which you are interested, and click on "eletters: Submit a response to this article" in the box in the top right hand corner. 